\title{
Classical eyelid border sign of neurofibromatosis
}

\author{
BYRON SMITH AND FRANK P. ENGLISH
}

From the Department of Ophthalmic Plastic Surgery, Manhattan Eye, Ear and Throat Hospital, New York

Neurofibromatosis is a well-documented entity, having been first recorded by von Recklinghausen (1882). When involvement of the orbit and eyelid occurs in this disease, the presentation may be manifold. Blepharoptosis commonly occurs and may be variable in degree. Where elephantiasis neuromatosa occurs in association with this, the drooping eyelid characteristically has the sensation of a bag of worms when palpated. In addition exophthalmos may or may not be present. Sometimes, where orbital bony defects exist, the globe has a pulsatile character; bone hypertrophy, however, may be the rule rather than bone destruction. The typical ocular and systemic manifestations of neurofibromatosis can also be associated with involvement of the orbit and these are well described in the literature (Reese, 1963).

Attention is drawn here to an important physical sign in the eyelid which is diagnostic of neurofibromatosis of the orbit. In this condition the lid margin of the ptotic eyelid displays a peculiar sinuous configuration, the lid border possessing an inner portion which arches upwards whereas the outer half assumes a downwards convexity (Fig. IA and B).

(IA)

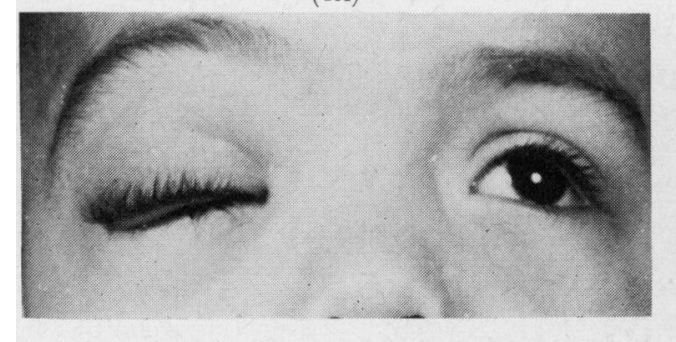

FIG. IA and B The sinuous eyelid border deformity of neurofibromatosis

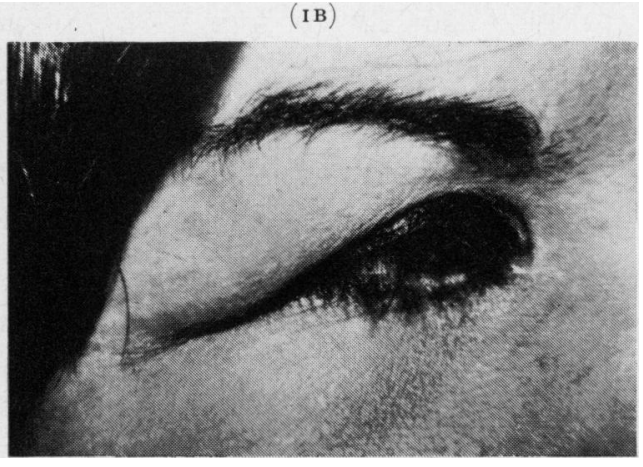

In considering the aetiology of this physical sign, a number of factors must be considered. The site of the tumour is important. Surgical exposure demonstrates the bulk of the tumour to be located predominantly lateral to the belly of the levator palpebrae superioris muscle; this accounts for the accentuation of the lid margin temporally. The tumour lies in close proximity to the conjunctiva and hence the transconjunctival route represents the proper surgical approach to these lesions. Attempts at removal via the skin are usually doomed to failure and the tumour is neither identified nor removed. The septum orbitale does not appear to play a role in this deformity, and may even exert a restraining influence as the septum is best developed in its lateral portion. 
Other important considerations include tarsal and integumental features. In neurofibromatosis the tarsal plate is abnormally pliable and loses its supportive role. This softening of the tarsal plate results in its distortion by movements of the eye. The same feature is sometimes seen in well-established trachoma (Herbert, 1907), when a similar change has occurred in the tarsal plate. The skin in neurofibromatosis is characterized by hyper-elasticity and can often be greatly stretched depending on the degree of involvement (Fig. 2); this loss of support is also partly responsible for the shape of the eyelid.

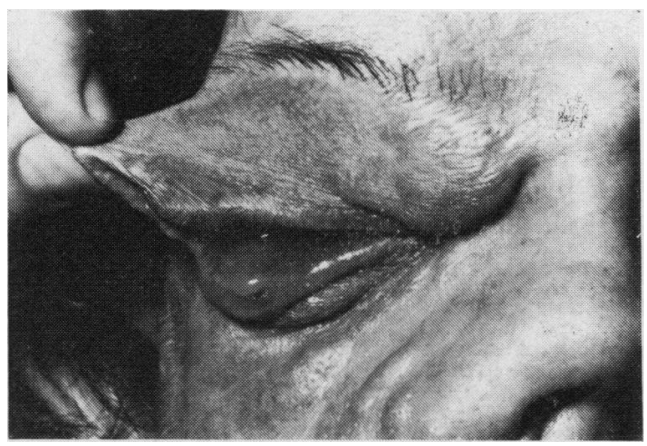

FIG. 2 Characteristic hyper-elasticity of the skin in neurofibromatosis

In the differential diagnosis, trachoma must be considered, as on rare occasions in advanced cases it can produce a similar clinical picture, but this is not as marked as in neurofibromatosis.

\section{Summary}

In neurofibromatosis of the orbit and eyelids, a sinuous deformity of the border of the eyelid is a common finding and is diagnostic of the condition.

\section{References}

HERBERT, H. (1907) Trans. ophthal. Soc. U.K., 27, $3^{8}$

RECKLINGHAUSEN, F. von (1882) "Ueber die multiplen Fibroine der Haut und ihre Beziehung zu den multiplen Neuromen". Hirschwald, Berlin

REESE, A. B. (1963) "Tumors of The Eye", 2nd ed., p. 194. Harper and Row, New York. 